\title{
Black Hole Shadow Observations with Space-Ground Interferometers
}

\author{
E. V. Mikheeva, S. V. Repin, V. N. Lukash \\ P.N. Lebedev Physical Institute of Russian Academy of Sciences \\ Profsoyuznaya 84/32, 117997, Moscow, Russia
}

April 23, 2020

\begin{abstract}
We consider the black hole $(\mathrm{BH})$ shadow images which can be restored by data processing and image recovery procedures in future space Very Large Baseline Interferometry (VLBI) missions. For Kerr BHs with masses and coordinates of $\mathrm{SgrA}^{*}, \mathrm{M} 87^{*}$ and M31*, illuminated by light source behind them, we consider three kinds of observation: the ground-based interferometer (similar to the Event Horizon Telescope), space-ground interferometer with a satellite in geocentric orbit and space-ground interferometer with a satellite located in Lagrange point $L_{2}$. The significant difference between the images produced by the ground-based telescope alone and the one of the space VLBI with an added low-orbit satellite is caused by both the increased baseline and the improved of $(u, v)$ coverage. The near-Earth configuration of the radio interferometer for the observation of $\mathrm{BH}$ shadows is the most preferable for the $\mathrm{BH}$ shadow observations among considered cases. With further increasing the orbit radius up to the Lagrange point $L_{2}$ the density of the $(u, v)$ filling decreases and the results appear less reliable. Model images for all the cases are presented.
\end{abstract}

\section{Introduction}

Nowadays the observation of the black hole $(\mathrm{BH})$ shadows is one of the hottest problems in the astrophysics (see review [1]-[3]). The pioneer observations of the BH shadow in the innermost area of M87 by the Event Horizon Telescope $(\mathrm{EHT})^{1}$ have just been made $\left.([4]-9]\right)$. The resulting images clearly demon-

\footnotetext{
${ }^{1}$ http://eventhorizontelescope.org
} 
strate the exceptional capabilities of modern instruments. At the same time, progress in the study of black holes and their nearby surroundings requires the continuation of research, i. e. the observation of $\mathrm{BHs}$ in other radio sources and at other (higher) frequencies.

At present, when the $\mathrm{BH}$ interferometry is developing very rapidly, the terms used to designate the BH images are: "shadow", "silhouette", and "photon ring" (the last term was first used in [10]). Sometimes their meaning is shared, but often mixed. So, in [11] the term "silhouette" is used, which is understood as the image of the BH event horizon, in [12] the term "shadow" is used, which refers to the image of the so-called photon sphere $(r=3 G M$ for a non-spinning BH). In English-language literature, terms "shadow" and "silhouette" are often confused. The authors follow this practice and name the shadow of $\mathrm{BH}$ its image for the specific model, without any detailing what the border of the shadow is. The image of the black hole depends both on its mass and rotation, and on the properties of the source that illuminates the black hole (disk, jet, bright spot, etc.). Some aspects of the building of the BH shadow in the General Relativity (GR) and extended theories of gravity have been discussed in [13] (see also references therein), as well as in [14]-[18].

However, regardless of the definition, the $\mathrm{BH}$ shadows have tiny angular sizes even for the closest BHs. This means that an VLBI technique should play a key role in shadow observations. Important parameters are also the magnitude of the baseline projection and the frequency at which the receiving equipment operates. The ground-based interferometers are limited by the Earth diameter and their baselines cannot exceed $12800 \mathrm{~km}$. However, the great advantage of this construction is that we can relatively easy control it, repair, develop and collect the big data arrays. The ground-based VLBI array is realized in EHT array, which has been carried out the first observations of the $\mathrm{BH}$ shadow in the center of M87 at $230 \mathrm{GHz}$.

An angular resolution of interferometer can be improved by increasing the baseline or by drifting to higher frequency. So, it would be a more productive idea to build the array of space-based radiotelescopes, which could form an interferometer with huge baselines ([19]-[21]). A more detailed consideration of possible satellite orbits can be found in [22]-[24]. The angular resolution of that interferometer might thousand times exceed the one for the groundbased devices. But, being realized, the mission appears to be very expensive and its technical support can hardly be realized as easy as it is executed for the ground-based antennas. In addition to the technical and financial problems there are a lot of difficulties in the filling of $(u, v)$ plane. In groundbased observations it is possible to achieve a relatively homogeneous coverage of the $(u, v)$ plane because the overall configuration of these tracks is almost 
the same every 24 hours, whereas in space-ground observations, especially with large projections of the base, the coverage is expected to be rather heterogeneous. It leads to the additional difficulties in imaging procedure.

Nevertheless, the idea of space-ground interferometer is very tempting and now it is under heat consideration and discussion (see [22]-224]). It can be realized in future space missions. One of them is the Millimetron space mission [25] with cooled 10-meter mirror operating in millimeter- and submillimeter bands, which is planned to be launched in late 2020s. The angular resolution of this instrument in the interferometric mode is assumed to be so high that we can clearly observe, in principle, the shadows of the BHs in many galaxies. However, there are reasons that worsen this ideal picture. For example, there are limitations on the sensitivity of the instrument, the radiation scattering by plasma inhomogeneities may occur, etc. In addition, all these effects depend drastically on the frequency. In the paper, we focus on the fundamental possibility of observing the shadow of a $\mathrm{BH}$ and neglect the nuances associated with the characteristics of specific observational instruments, as well as specific astronomical objects. The preliminary catalog of supermassive BHs can be found in [26, where the observational possibilities of Millimetron mission were taken into account. The catalog is based on the extended catalog [27].

The interpretation of the interferometric observations of $\mathrm{BH}$ shadows requires the simulation of the expected image. This problem, in turn, requires a lot of effort to develop the radiation source models. As it is shown in many papers ([2], [28]-36]) the $\mathrm{BH}$ image depends significantly on the $\mathrm{BH}$ surrounding, i. e. on the structure of the accretion disk, the dependence of its temperature on the radial coordinate, on the existence of relativistic plasma jet, etc. It is also necessary to take into account the radiation of the corona, the geometry of the magnetic field, the presence of synchrotron radiation, and more. Except that, the image depends also on the interstellar scattering processes ([37, 38]). In our simulation we do not take into account all these effects and use the simplest model of the shadow image, which depends only on the mass and spin of a $\mathrm{BH}$ and the source of photons - the bright plane behind the BH. Nevertheless, it allows us to reveal the characteristic features of the results of ground-based observations and the observations in space-ground interferometer. We do not consider an image of the real source, SgrA* (or M87*, M31*), but a model of a BH shadow with the same angular size.

The recent observation of the $\mathrm{BH}$ shadow was provided by the groundbased interferometer. This means that we need to study the prospects for future research of BHs. The most evident way to do it is to join the experiences of EHT and RadioAstron mission [39] in future space experiments. In 
the paper we compare the images of the $\mathrm{BH}$ shadow which can be obtained by the interferometers with different configurations.

Main goal of the paper is to discuss the preferred satellite orbit, which should allow to obtain the shadow of a $\mathrm{BH}$ with high resolution quickly. Such a high-quality image is able to deliver the important information about the physical processes in the very vicinity of supermassive BHs (structure of the inner disk and base of a plasma jet), inhabiting the innermost parts of massive galaxies. This also can be applied to test the General Relativity in strong gravitational fields. We do not discuss the technical problems of interferometry and leave their solution to VLBI specialists.

\section{Model of BH shadow}

We consider the spinning $\mathrm{BH}$ and its shadow, or silhouette, with a simple geometry of photons source. We assume that a $\mathrm{BH}$ is described by the Kerr metric and its spin is close to maximal, $a=0.9981$ (dimensionless parameter describing the ratio of the angular momentum of the $\mathrm{BH}$ to its mass). The spin axis is perpendicular to the view line of the distant observer (see fig. 1). Behind the $\mathrm{BH}$ and far away there is a bright flat screen, which emits the quanta uniformly to a hemisphere (in solid angle $2 \pi$ ). If the screen plane is perpendicular to the view line of the distant observer then the BH silhouette looks like the one shown in fig. 22. The similar image might appear if a BH of the stellar mass orbits a red giant star and passes in front of the star.

To build the photon trajectories we solved the equations of motion under the General Relativity assumptions for each quantum. The system of six ordinary differential equations can be found in [40, 41]. Ordinary differential equation solvers are included in many packages and freely distributed in Internet $\left([42,43]^{3}\right)$. The simulated image counts the trajectories of approximately 10 million quanta.

This image has got a number of characteristic details: it is asymmetric, its brightness is inhomogeneous and, finally, it includes the thin annuli inside, formed by the quanta, which came to the observer after a few revolutions around the BH. The left hand side of the annuli is actually presents, but cannot be adequately displayed because the width of all the annuli is much smaller than the pixel diameter. On the right hand side the annuli can be seen separately and their total width is only 17-18 times less than the shadow

\footnotetext{
${ }^{2}$ If the BH was irradiated from a solid angle $4 \pi$, the shadow image would look different: a wide dark ring between narrow photonic arcs and a contrasting edge (the red ring in the color system fig. 2 would be light.

${ }^{3}$ https ://www.mcs.anl.gov/petsc/
} 




Figure 1: The location of the emitting surface, the BH and the observer. The view line of the observer is perpendicular to both the screen plane and the spin axis of the $\mathrm{BH}$.

diameter. Thus we consider the image of a $\mathrm{BH}$ shadow, which has enough small size details to elaborate and discuss the data processing technique. Certainly, such small and dim details will be highly likely blurred by the scattering processes, but this fact will be considered later (see Conclusion).

We presume that the spin axis of the $\mathrm{BH}$ in the center of the Milky Way is perpendicular to the galactic plane and coincides with the axis of the Milky Way rotation. It means, that we know its orientation on the celestial sphere. As to M87 and M31, their spin axes and the spin axes of their BHs keep some uncertainty yet, so we accepted that their spins are oriented along the declination axis. Finally, we assume that our sources are time-independent.

\section{$3 \quad$ Images of $\mathrm{BH}$ shadows}

To reconstruct the images we used the well-established CLEAN procedure $([44,45])$. This algorithm is widely used in astrophysics and allows to extract the image from the Fourier coefficients on a finite number of $(u, v)$ plane points (which implies a smoothing procedure). Mathematically, we deal with an incorrect problem because a coverage of $(u, v)$ plane is incomplete. As it has been shown, for example, in [7], the BH shadow images have some deviations between different image recovery methods and their different implementations. Nevertheless, as it has been demonstrated there, the morphology of images remains unchanged. In the paper, we also focus on the morphology of the image and do not consider the features that may be associated with the use of a specific procedure of image recovery or with the source model. The CLEAN method does give a general idea of the shadow image. 


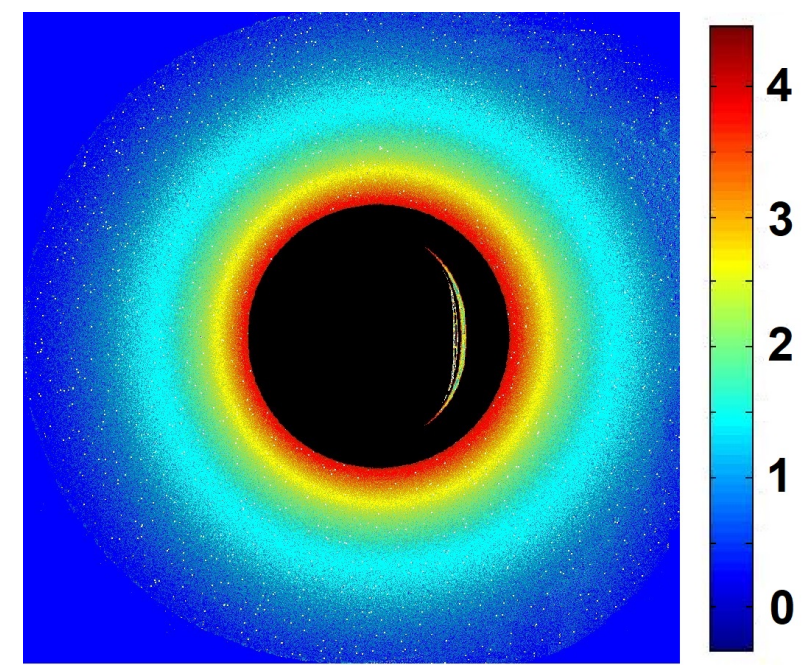

Figure 2: The shadow of a Kerr black hole against the background of a flat luminous screen perpendicular to the line of sight of distant observer. The black hole spin axis is also perpendicular to the line of sight. The intensity is presented in logarithmic scale and normalized by the brightness of the background screen.

\subsection{Ground-based Interferometer}

First, we consider the image, which can be reconstructed after the groundbased interferometric observations like the ones carried out by EHT. The daily coverage of $(u, v)$ plane for $\operatorname{SgrA}^{*}$ shown in fig. 3 on the top left panel. The coverage of $(u, v)$ plane shown in the Figure is just a possible example and does not coincide with the real observational set of the objects by EHT. The maximal base projection here is about $0.8 R_{\odot}$. For other objects the look of $(u, v)$ plane coverage may vary due to the different celestial coordinates, but the general view remains approximately the same. As it follows from the Figure the coverage is dense enough and looks relatively uniform.

Three other panels in fig. 3 present the images of the $\mathrm{BH}$ shadow from fig. 2 observed at the frequency of $240 \mathrm{GHz}$ and reconstructed then by CLEAN technique for coordinates and masses of $\mathrm{SgrA}^{*}, \mathrm{M}^{*} 7^{*}$ and M31*. As it follows from the Figure the resolution of the image is not high, but we can identify some details of the characteristic image details, especially for SgrA*. For example, at the top right corner there is a bright detail which can be interpreted as a narrow crescent, which can also be found in the original model image. On the restored images of other BH models (bottom panels of fig. 3), the shadow is not visible. Asymmetry and heterogeneity of the image are an artifact of the data processing procedure. 

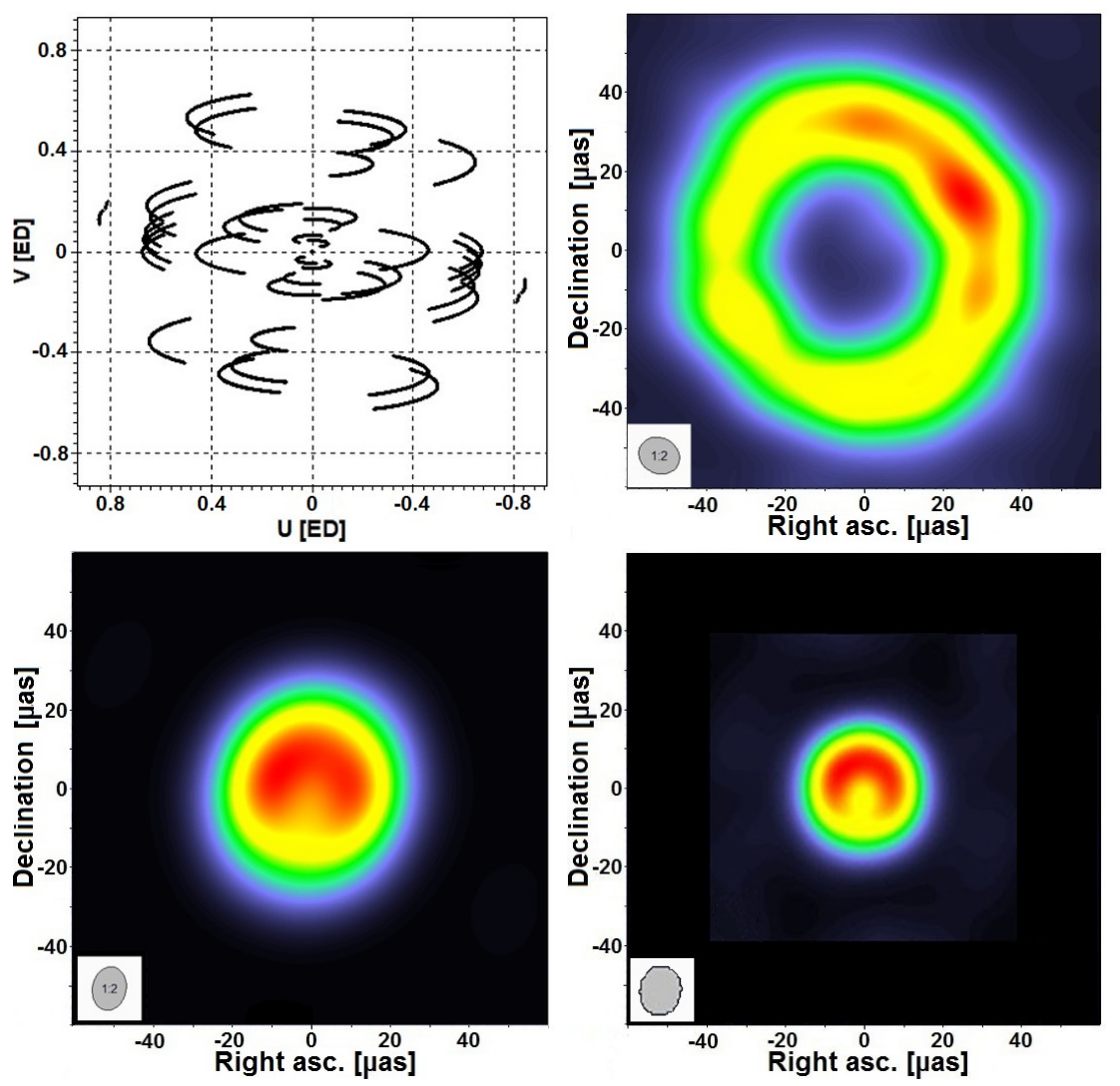

Figure 3: Images of a sample BH shadow for $\mathrm{SgrA}^{*}$ (the top right panel), M87* (bottom left) and M31* (bottom right) restored by data processing for ground-based interferometric observations. The appropriate coverage of $(u, v)$ plane is presented for SgrA*. The images are shown in conditional colors.

\subsection{Low-orbit satellite Interferometer}

The low-orbit interferometer implies the satellite at the orbits at 200-300 $\mathrm{km}$ from the Earth to the geostationary orbit. Their mean value radius is approximately $2 \div 3 R_{\oplus}$. Each satellite in the array makes from 1 to 16 revolutions around the Earth per day, depending on the radius. In our simulation the radius of the orbit is close to $2 R_{\oplus}$. The daily coverage of $(u, v)$ plane for SgrA* $^{*}$ is shown on the top left panel in fig. 4. The tracks of ground-based telescopes are also presented in fig. 4, they are the same as those shown in fig. 3, but in a reduced scale. As it has been above mentioned the general view of the coverage is approximately the same for M87* and M31*.

The images of the objects, restored by the CLEAN technique, are shown on three other panels in fig. 4. The top right panel presents the image of 

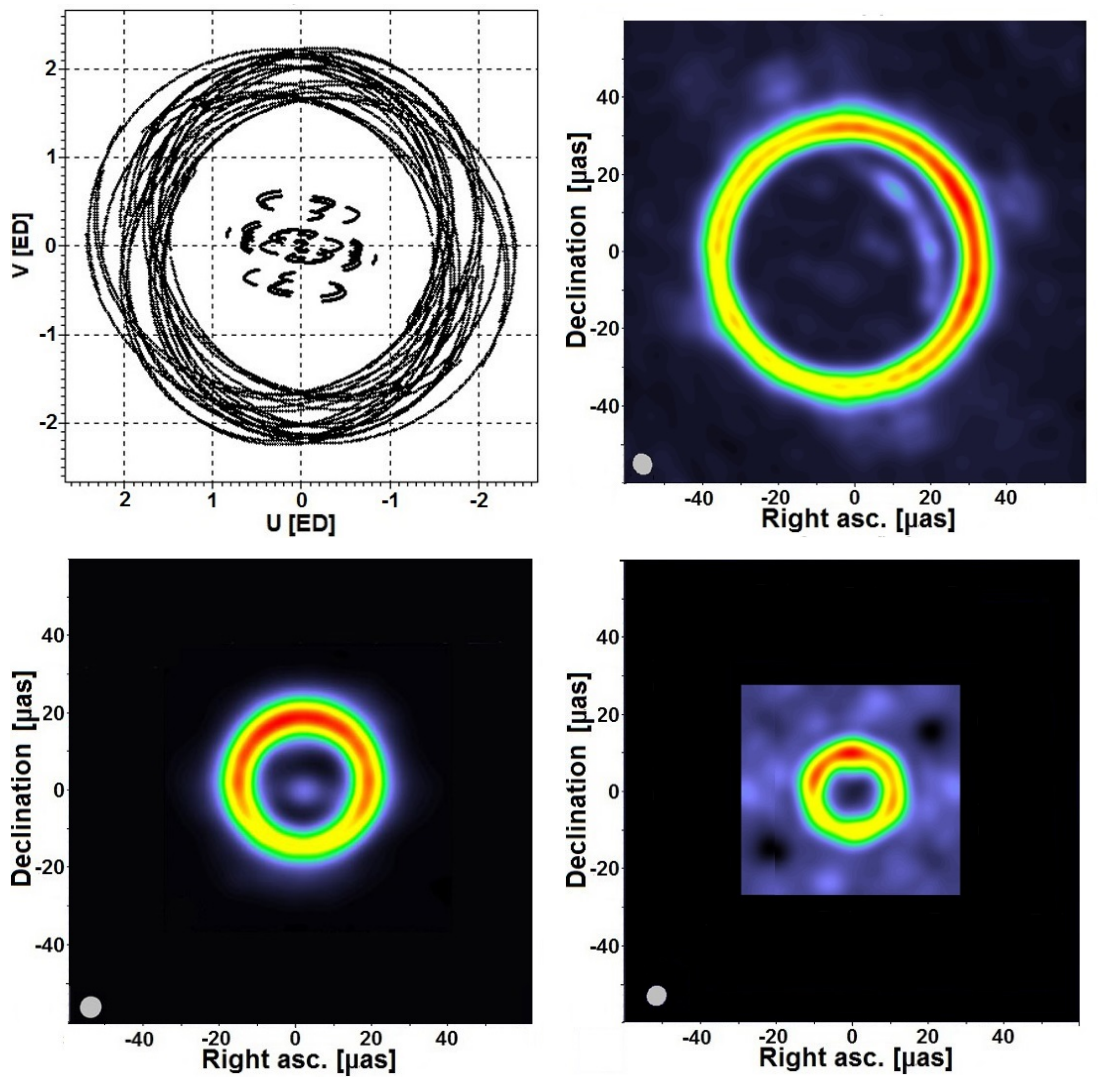

Figure 4: Images of a sample BH shadow for SgrA* (the top right panel), M87* (bottom left) and M31* (bottom right) restored by data processing for space-ground interferometer with a satellite at low geocentric orbit. The appropriate coverage of $(u, v)$ plane is presented for SgrA*. The images are shown in conditional colors.

SgrA*. This image contains a lot of additional details in comparison with fig. 3. Thus, in the top right part of the inner dark area one can see the bright crescent that corresponds to the appropriate detail in fig. 2. Moreover, we can even guess that this image is rotated at some angle compared to fig. 2 and measure immediately this angle. It gives us the important information on the momentum of the $\mathrm{BH}$ and the direction of its axis. It means that the angular resolution in this case is so high that after the image processing we can examine in detail the parameters of a $\mathrm{BH}$ and its environment. The bright circle has also the inhomogeneous intensity distribution and the position of its maximum is in agreement with the one of the inner crescent.

The images of $\mathrm{M} 87^{*}$ and $\mathrm{M} 31^{*}$ on the bottom panels in fig. 4, have also additional characteristic features. It is clearly seen that the intensity of the 

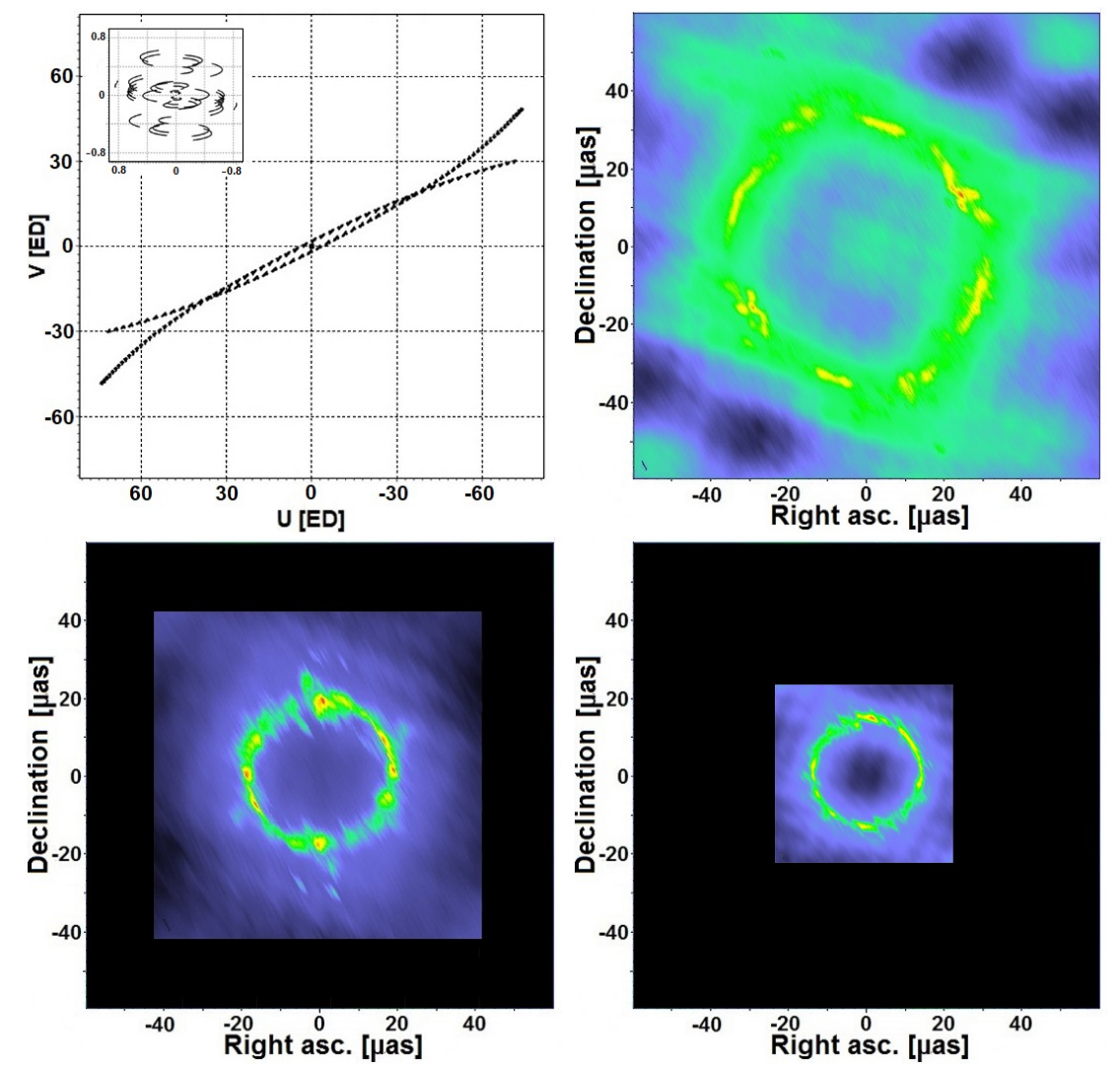

Figure 5: Images of a sample BH shadow for $\mathrm{SgrA}^{*}$ (the top right panel), M87* (bottom left) and M31* (bottom right) restored by data processing space-ground interferometer with a satellite located in Lagrange point $L_{2}$. The appropriate coverage of $(u, v)$ plane is presented for SgrA*. The images are shown in conditional colors.

bright ring is inhomogeneous and this inhomogeneity carries the important information about the orientation of the BH spin axis. Higher angular resolution leads us to the fact that both the range of intensity in the images and the intensity gradients are much higher than in fig. 3

The comparison of the images in fig. 3 and 4 allows us to draw a conclusion that the low orbital space-ground interferometer, being realized, can get us much more information about the nature of the BHs than the observations of the ground-based interferometers only. 


\subsection{High-orbit Satellite Interferometer}

The next step in increasing the baseline would be placing the satellite in the Lagrangian point $L_{2}$, that is the case of the planning space observatory Millimetron. After the satellite reaches this point it remains there during all the experiment time. The interferometer base can be really huge and its projection for some sources may exceed $100 R_{\oplus}$. However, the coverage of $(u, v)$ plane becomes degenerated. Except that during the 5-year experiment one can hope for only a single observation of a specific object.

As before, the coverage of $(u, v)$ plane in this case for $\operatorname{Srg} \mathrm{A}^{*}$ is shown on the top left panel in fig. 5. The tracks of the ground-based antennas cannot be shown adequately because of the fig. 5 scale. But they are really present in the very center of this Figure. They are also presented in the inset. Notice that the low-orbit satellite is not included in the consideration here. The other panels in fig. 5 demonstrate the reconstructed images of SgrA*, M87* and M31*.

The angular resolution of this configuration is extremely high, but because of very poor coverage of the $(u, v)$ plane the result looks worse than in fig. 4 . The main reason is that this coverage of the $(u, v)$ plane does not permit to restore reliably the original image.

The bright photon ring can be easily distinguished in the image of $\mathrm{SgrA}^{*}$, but despite of the very high angular resolution, the narrow inner crescent detail is completely lost, so any information about the axis orientation is lost too. The intensity distribution along the ring looks also uniform, in contrast to fig. 4.

Analysis of the images of the considered $\mathrm{BH}$ shadow model with M87* and M31* coordinates reveals the same features. We can conclude only that a certain ring-like structure is definitely observed. The edge of the shadow in M87* also does not reveal any characteristic details that would allow to establish the direction of the angular momentum.

\section{Conclusions}

We found that the interferometer, which includes both the ground-based telescopes and a low-orbit satellite has the advantage in comparison with other reviewed cases. The key role here plays an ability to fill the $(u, v)$ plane with high density during the relatively short time (a day or week). And a low-orbit interferometer is able to successfully solve this problem because the good coverage of the $(u, v)$ plane takes here less than a week. Moreover, if the orbit lays at about 300-400 km above the Earth the revolution lasts 1.5 hour 
and after 5-7 cycles the coverage of the $(u, v)$ plane becomes dense enough. At that case the observation procedure may last only about 10 hours and, in principle, we can get the $\mathrm{BH}$ image with satisfactory quality and resolution twice a day. A satellite in the orbit with $R \sim 2 R_{\oplus}$ has about three times longer orbital period, so a similar coverage of the $(u, v)$ plane will be achieved in about a day (see fig. 4). The simultaneous use of two or three satellites can further reduce this time.

The ground-based arrays have an enough $(u, v)$ coverage, but the most objects cannot be observed all day long because they are below the horizon during some period. At those periods the coverage of $(u, v)$ plane is, certainly, stopped and we have the traces which look like a half of ellipse (see fig. 3). The great advantage of ground-based instruments is, obviously, their low cost.

The longer is a baseline of the interferometer the more difficult is the correlation process. In particular, this is due to the difficulty in synchronizing of ground and on-board timers. It means that the space-ground interferometer should have much more sophisticated equipment than a ground instrument.

A case of a high-orbit satellite differs from others. Indeed, its angular resolution is incredibly high. Theoretically, it is so high that we would be able to see the annuli inside the shadow area in fig. 2 separately, i. e. the resolution could be even greater than it is reproduced in the Figure. However, we face here a problem with a poor $(u, v)$ plane coverage. The fact is that the information obtained in the interferometric observation is not the image of the object itself. It is a Fourier Transform of the true image at limited number points of the $(u, v)$ plane. The reliable image reconstruction is possible only with a dense enough coverage of $(u, v)$ plane. One turn around the Earth of the satellite in Lagrangian point $L_{2}$ takes a full year. And even an increase in the duration of observations will not have a radical effect on the coverage of the $(u, v)$ plane. A spacecraft moving in the orbit near point $L_{2}$ every year almost repeats its track on the $(u, v)$ plane. The displacement of the spacecraft up and down from the ecliptic plane only increases slightly the coverage of the $(u, v)$ plane.

Another interesting idea is to place the radiotelescope at the Lunar pole [21]. The orbital period of the natural Earth satellite lasts about a month, so one third or a half of a year (a few turns around) is enough to cover the $(u, v)$ plane. In any case, this is much less than that is required for the satellite located at the Lagrange point $L_{2}$. However, for the implementation of such a colossal project there are still many technical problems to be solved.

At the present only two successful space VLBI missions have been implemented: the VLBI Space Observatory Programme (VSOP) [46] and RadioAstron [39]. Both were equipped by the receivers in centimeter wave bands, so 
it means that we have not enough experience yet.

The long-awaited detections of the BH shadow by EHT is the first step to test the General Relativity in strong gravitational fields. However, one has found that its quantitative features are not sufficient to distinguish between BHs using different theories of gravity. It is highlighting the fact that the great caution is needed when interpreting the $\mathrm{BH}$ images as the tests of General Relativity [47. Since the BH shadow can be measured more precisely by the space-ground interferometer than by the ground-based one, the spaceground VLBI mission does allow to carry out the stronger tests of the General Relativity and the accretion models.

\section{Financial support}

This activity has been partly supported by Russian Foundation for Basic Research, grant 19-02-00199 and the RAS project KP 19-270 "Questions of the origin and evolution of the Universe using methods of ground observations and space research".

\section{Acknowledgement}

The authors are grateful to Dr. A. Andreanov for his kind help in preparation of the BH shadow images and numerous useful discussion. We are grateful to Dr. V.N. Strokov Dr. I.N. Pashchenko for their help with the manuscript preparation. We also grateful to Dr. V.I. Kostenko and Dr. S.F. Likhachev for useful discussions. One author (S.R.) is very grateful to Dr. O.N. Sumenkova, Dr. R.E. Beresneva and Dr. O.A. Kosareva for the possibility of fruitful working on this problem.

\section{References}

[1] H. Falcke, J. Physics: Conference Series 942, id. 012001 (2017).

[2] J.-P. Luminet, arXiv:1804.03909v1 (2018).

[3] S. Nampalliwar and C. Bambi Tutorial Guide to X-ray and Gamma-ray Astronomy: Data Reduction and Analysis, edited by C. Bambi (Singapure: Springer, 2019), (arXiv:1810.07041v3).

[4] Event Horizon Telescope Collaboration: K. Akiyama, A. Alberdi, W. Alef, K. Asada, et al., Astrophys. J. 875, id. L1 (2019). 
[5] Event Horizon Telescope Collaboration: K. Akiyama, A. Alberdi, W. Alef, K. Asada, et al., Astrophys. J. 875, id. L2 (2019).

[6] Event Horizon Telescope Collaboration: K. Akiyama, A. Alberdi, W. Alef, K. Asada, et al., Astrophys. J. 875, id. L3 (2019).

[7] Event Horizon Telescope Collaboration: K. Akiyama, A. Alberdi, W. Alef, K. Asada, et al., Astrophys. J. 875, id. L4 (2019).

[8] Event Horizon Telescope Collaboration: K. Akiyama, A. Alberdi, W. Alef, K. Asada, et al., Astrophys. J. 875, id. L5 (2019).

[9] Event Horizon Telescope Collaboration: K. Akiyama, A. Alberdi, W. Alef, K. Asada, et al., Astrophys. J. 875, id. L6 (2019).

[10] M. D. Johnson, A. Lupsasca, A. Strominger, G. N. Wong, et al., arXiv:1907.04329v2.

[11] V. I. Dokuchaev, N. O. Nazarova, V. P. Smirnov, General Relativ. and Gravit. 51, id. 81 (2019).

[12] V. Perlick, O. Yu. Tsupko, G. S. Bisnovatyi-Kogan, Phys. Rev. D 92, id. 104031 (2015).

[13] A. F. Zakharov, Phys. Rev. D 90, id. 062007 (2014).

[14] A. F. Zakharov, Intern. J. Modern Physics D 27, id. 1841009 (2018).

[15] V. Perlick, O. Yu. Tsupko, G. S. Bisnovatyi-Kogan, Phys. Rev. D 97, id. 104062 (2018).

[16] S. O. Alekseyev, V. A. Prokopov, E. D. Emtsova, B. N. Latosh, JETP 128(5), 720 (2019).

[17] V. I. Dokuchaev, N. O. Nazarova, arXiv:1911.07695v2 (2020).

[18] S. O. Alekseyev, V. A. Prokopov, arXiv:2001.09272 (2020).

[19] L. I. Matveenko, N. S. Kardashev, G. B. Sholomitskii, Radiophysics and Quantum Electronics, 8, 461 (1965).

[20] F. Roelofs, H. Falcke, C. Brinkerink, M. Moscibrodzka, Astron. and Astrophys. 625, id. A124 (2019).

[21] T. An, X. Hong, W. Zheng S. Ye et al., Adv. Space Res. 65(2), 850 (2020). 
[22] V. L. Fish, M. Shea, and K. Akiyama, Adv. Space Res. 65(2), 821 (2020).

[23] T. Kawashima, M. Kino, and K. Akiyama, Astrophys. J. 878(1), id. 27 (2019).

[24] D. C. M. Palumbo, S. S. Doeleman, M. D. Johnson, K. L. Bouman, and A. A. Chael, Astrophys. J. 881(1), id. 62 (2019).

[25] N. S. Karddashev, I. D. Novikov, V. N. Lukash, S. V. Pilipenko, et al., Phys. Usp. 57, 1199 (2014).

[26] P. B. Ivanov, E. V Mikheeva, V. N. Lukash, A. M. Malinovsky, et al., Phys. Usp. 62, 423 (2019).

[27] E.V. Mikheeva, V.N. Lukash, S.V.Repin, and A.M. Malinovskii, Astron. Rep. 63, 343 (2019).

[28] J.-P. Luminet, Astron. and Astrophys. 75, 228 (1979).

[29] H. Falcke, F. Melia, and E. Agol, Astrophys. J. 528, L13 (2000).

[30] J. Dexter, J. C. McKinney, and E. Agol, Monthly Not. Roy. Astron. Soc. 421, 1517 (2012).

[31] L. Amarilla and E. F. Eiroa 14 Marcel Grossman Meeting (Singapure: World Scientific, 2017), 3543.

[32] A. Abdujabbarov, M. Amir, B. Ahmedov, and S. G. Ghosh, Phys. Rev. D 93, id. 104004 (2016).

[33] A. E. Broderick, V. L. Fish, M. D. Johnson, K. Rosenfeld, et al., Astrophys. J. 820(2), id. 137B (2016).

[34] M. Moscibrodzka, H. Falcke, and H. Shiokawa, Astron. and Astrophys. 586, id. A38 (2016).

[35] A. Neronov and I. Vovk, Phys. Rev. D 93, id. 023006 (2016).

[36] T. Johannsen, A. E. Broderick, P. M. Plewa, S. Chatzopoulos, et al., Phys. Rev. Lett. 116, id. 031101 (2016).

[37] Z. Zhu, M. D. Johnson, R. Narayan, Astrophys. J. 870, id. 6 (2019).

[38] M. D. Johnson, R. Narayan, D. Psaltis, L. Blackburn, et al., Astrophys. J. 865(2), id. 104 (2018). 
[39] N. Kardashev, V. Khartov, V. Abramov, V. Yu. Avdeev, et al., Astron. Rep. 57(3), 153 (2013).

[40] A. F. Zakharov and S. V. Repin, Astron. Rep. 43(1), 705 (1999).

[41] S. V. Repin, D. A. Kompaneets, I. D. Novikov, V. A. Mityagina, arXiv:1802.04667 [gr-qc] (2018).

[42] L. R. Petzold, SIAM J. Sci. Comp. 4(1), 136 (1983).

[43] S. Abhyankar, J. Brown, E. M. Constantinescu, D. Ghosh, B. F. Smith, and H. Zhang, arXiv:1806.01437 [mathNA] (2018).

[44] J. A. Hogbom, Astron. and Astrophys. Suppl. Ser. 15, 417 (1974).

[45] U. J. Schwarz, Astron and Astrophys. 65, 345 (1978).

[46] H. Hirabayashi, H. Hirosawa, H. Kobayashi, Y. Murata, et al., Science 281(5384), 1825 (1998).

[47] Y. Mizuno, Z. Younsi, C. M. Fromm, and O. Porth, Nature Astron. 2, 585 (2018). 